\title{
Perfil Antropométrico de Jugadoras Chilenas de Fútbol Femenino
}

\author{
Anthropometric Profile of Female Football - Soccer Chilean Players \\ *Atilio Aldo Almagià Flores; "Fernando Rodríguez Rodríguez; "Fernando Omar Barrraza Gómez; \\ "Pablo José Lizana Arce \& **Carlos Alberto Jorquera Aguilera
}

ALMAGì̀, A. F.; RODRÍGUEZ, R. F. J.; BARRAZA, G. F. O.; LIZANA, P. J. \& JORQUERA, A. C. A. Perfil antropométrico de jugadoras chilenas de futbol femenino. Int. J. Morphol., 26(4):817-821, 2008.

RESUMEN: Se evaluó un total de 43 jugadoras de fútbol, 26 seleccionadas chilenas sub 20 y 17 jugadoras del plantel campeón de fútbol universitario 2007 de la Pontificia Universidad Católica de Valparaíso (PUCV), todas ellas bajo su consentimiento informado. Se evaluó utilizando el protocolo de marcaje y medición de la International Society for the Avancement in Kineanthropometric (ISAK), en condiciones normales de temperatura, a primera hora de la mañana y después del vaciado urinario, por evaluadores con licencia nivel II de ISAK. Las variables medidas fueron peso, estatura de pie, estatura sentada, perímetros musculares, diámetros óseos y pliegues cutáneos. Todas estas medidas se utilizaron para las fórmulas de composición corporal de Kerr (1988) y para el método antropométrico del somatotipo de Heath-Carter (2004). Existe una diferencia de 3,4 años entre ambos grupos, lo que diferencia algunos otros componentes de la morfoestructura, como una mayor cantidad de grasa y menor cantidad de músculo del grupo sub 20 en comparación con el grupo PUCV y una representación grafica del somatotipo que corrobora estas diferencias. Las diferencias en los componentes de la composición corporal entre ambos grupos tienen dos razones fundamentales. La primera por el mayor desarrollo y maduración física del grupo PUCV el cual al ser mayor, presenta promedios de estatura y peso también mayores. La segunda razón es el nivel de entrenabilidad del grupo PUCV que, al tener un mayor desarrollo, permite la realización de entrenamientos más prolongados y de mayor intensidad, demostrando un gran desarrollo muscular y menor cantidad de grasa en comparación con el grupo sub-20. Concluimos que según los factores antropométricos, el grupo PUCV es capaz obtener un rendimiento deportivo mayor que el grupo sub-20, siendo esto un importante fundamento al momento de elegir a las jugadoras pre-seleccionadas nacionales.

PALABRAS CLAVES: Somatotipo; Composición Corporal; Antropometría; Fútbol Femenino.

\section{INTRODUCCIÓN}

El fútbol femenino ya ha superado los 40 millones de jugadoras en el mundo en 15 años, sobrepasando así al fútbol juvenil masculino. $\mathrm{Y}$ es que la cifra anterior no es menor, sobre todo si se piensa que son casi 120 millones los jugadores de todas las categorías que componen hoy el fútbol masculino.

Si bien en Europa es practicado desde los años setenta, el primer puntapié del fútbol femenino fue dado por las noruegas en el Congreso de la Fedération internationale de Football Association (FIFA) del año 1986, en México, cuando se disputó el Mundial Masculino, donde llamaron la atención del entonces presidente de la FIFA, el brasileño João Havelange, quien accedió a la realización de un torneo experimental que tuvo lugar en 1990, en China (FIFA, 2004).

El éxito fue tal, que al año siguiente se dispuso la conformación de la Copa Mundial de Fútbol Femenino de la FIFA con periodicidad de 4 años impares, un año posterior al mundial de fútbol masculino.

De esta manera, las mujeres se han ido posicionando poco a poco en la práctica del fútbol, incorporándose a categorías menores, sub17 y sub 20 y aumentando en nuestro país las competencias locales, regionales y universitarias.

Al mismo tiempo del desarrollo de más competencias, aumentas las exigencias físicas que favorezcan una mejora del rendimiento deportivo, considerando entrenamientos para el aumento de la capacidad aeróbica, capacidad de salto y velocidad, forzando a las deportistas a producir cambios en la distribución de los componentes de la morfoestructura.

El mundial de fútbol femenino sub 20 en Chile ha suscitado gran atención sobre los factores que podrían mejorar el rendimiento físico y los cambios estructurales de las

\footnotetext{
* Laboratorio de Antropología Física y Anatomía Humana, Instituto de Biología, Facultad de Ciencias, Pontificia Universidad Católica de Valparaíso, Chile.

***Escuela de Nutrición y Dietética, Facultad de Medicina, Universidad Mayor, Chile.
} 
jugadoras. Conociendo la relación entre algunos componentes de la condición física y la morfoestructura, son analizados y discutidos los factores antropométricos de jugadoras de fútbol de Chile.

\section{SUJETOS Y MÉTODO}

Fue evaluado un total de 42 jugadoras de fútbol, 25 seleccionadas chilenas sub 20 y 17 jugadoras del plantel campeón 2007 de fútbol universitario de la Pontificia Universidad Católica de Valparaíso (PUCV), todas ellas fueron evaluadas bajo su consentimiento informado.

Las mediciones se realizaron bajo el protocolo de marcaje y medición de la International Society for the Avancement in Kineanthropometric (ISAK), en condiciones normales de temperatura, a primera hora de la mañana y después del vaciado urinario. Los evaluadores tenían licencia nivel II de la ISAK.

Las variables evaluadas fueron: peso, estatura de pie, estatura sentado, perímetros musculares, diámetros óseos y pliegues cutáneos. Todas estas medidas se utilizaron para las fórmulas seleccionadas en nuestro estudio. Composición corporal de Dhebora Kerr (Kerr, 1988), el cual es un método pentacompartimental, que divide la morfoestructura en masa grasa, masa muscular, masa ósea, masa residual y masa de la piel. Menor cantidad de variables se utilizan para el método antropométrico del Somatotipo de Heath-Carter (Carter, 2004), el cual es un método tricompartimental que divide la morfoestructura en masa muscular, masa ósea y masa grasa, de acuerdo a la forma corporal. Para dichas medidas y protocolo se usó un kit antropométrico Rosscraft SRL validado por la ISAK, que incluye antropómetros óseos, huincha métrica y plicómetro o calibre para pliegues.

Para establecer las diferencias entre los resultados antropométricos de ambos grupos, primero se realizó un análisis descriptivo de las variables de interés: peso, talla, índice de masa corporal (IMC), masa grasa, muscular y somatotipo.

Una vez analizados descriptivamente los resultados se llevó a cabo un test ANOVA de un factor, cuando las variables cumplían los supuestos de normalidad y homocedasticidad y un test de Kruskal-Wallis, para aquellas que no cumplían estos supuestos. Este último contraste fue el que aplicamos ya que no cumplían con las condiciones de aleatoridad, debido a las características de muestra como grupo modelo. Al igual que las demás técnicas no paramétricas, la de Krustal-Wallis, se apoya en el uso de los rangos asignados a las observaciones.

\section{RESULTADOS}

El grupo de elite del fútbol femenino chileno, tiene un promedio de edad joven, El IMC se encuentra dentro del rango que la Organización Mundial de la Salud (OMS) considera como normal, aunque pocas veces se considera como una medida confiable, debido a que suma el peso de la masa muscular de los deportistas para el cálculo total del peso, pero que consideramos mostrar como una forma viable de comparación.

De a cuerdo a los anterior, las deportistas presentaron una alta masa muscular, pero también alta masa grasa, que también se expresó en la sumatoria de pliegues (Tabla I).

Una vez llevado a cabo los diferentes protocolos de recogida de datos y aplicadas las distintas pruebas estadísticas, se presentan en la Tabla I, los valores de las medidas antropométricas de todas los jugadoras y sus respectivos equipos, indicando que no existen diferencias importantes entre los grupos en la mediana y el promedio. Sin embargo, al observar las desviaciones estándar, el equipo PUCV es más heterogéneo en los porcentajes de los cinco componentes de la composición corporal. Aunque el porcentaje (\%) de masa muscular es inferior al equipo sub 20 .

Al prestar atención al somatotipo, tampoco se vislumbran diferencias entre los grupos.

A modo de obtener una mejor percepción de los grupos, se realizó un análisis gráfico (boxplot) de las variables de interés. Los resultados se presentan a continuación:

En la Fig. 1, se puede observar que el equipo PUCV ttenía una mayor dispersión en cuanto al \% de masa adiposa que el equipo sub 20. Ambos equipos presentaban medianas parecidas, sólo diferían en la variabilidad.

En cuanto al \% de masa muscular, es apreciable que PUCV tenía una mediana superior a la del equipo sub 20.

La Fig. 2, no evidencia diferencia significativa entre los somatotipos medios de las jugadoras de ambos equipos. Pues las medidas de tendencia central como la dispersión fueron similares. Se puede afirmar que los dos grupos deportivos eran homogéneos en su somatotipo.

En la Fig. 2, los valores "p" de cada variable, indican que sólo el porcentaje de masa muscular era estadísticamente diferente entre los grupos. El equipo PUCV presentaba un $\%$ masa muscular significativamente superior al del equipo sub 20 . 
Tabla I. Resultados de composición corporal, sumatoria de 6 pliegues, índice de masa corporal (IMC) y somatotipo de las individuos utilizados en el estudio.

\begin{tabular}{|c|c|c|c|c|c|c|c|c|c|c|}
\hline 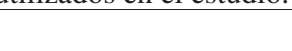 & \multicolumn{5}{|c|}{ SELECCIÓN Sub 20} & \multicolumn{5}{|c|}{ SELECCIÓN PUCV } \\
\hline & PROM & D ST & MEDNA & MIN & MÁX & PROM & D ST & MEDNA & MIN & MÁX \\
\hline Edad (años) & 18,1 & 0,7 & 18,1 & 16,9 & 19,4 & 21,5 & 1,9 & 21,0 & 20,0 & 27,0 \\
\hline Peso (kg) & 59,7 & 6,4 & 59,0 & 51,9 & 78,3 & 62,1 & 8,8 & 62,0 & 50,4 & 81,0 \\
\hline Talla $(\mathbf{c m})$ & 159,2 & 5,0 & 160,0 & 151,0 & 168,0 & 162,6 & 5,0 & 163,0 & 153,0 & 171,0 \\
\hline Talla sentado $(\mathrm{cm})$ & 83,6 & 2,1 & 84,1 & 79,5 & 88,0 & 86,2 & 2,6 & 86,0 & 81,0 & 90,0 \\
\hline Sum 6 pliegues $(\mathrm{mm})$ & 82,5 & 29,1 & 79,0 & 6,7 & 141,0 & 86,5 & 30,6 & 81,0 & 50,0 & 141,0 \\
\hline$\%$ piel & 5,6 & 0,5 & 5,8 & 4,8 & 6,3 & 5,2 & 0,9 & 5,3 & 2,3 & 6,1 \\
\hline$\%$ Masa adiposa & 29,1 & 2,3 & 29,0 & 23,8 & 33,6 & 28,4 & 4,1 & 27,9 & 23,1 & 36,4 \\
\hline \% Masa muscular & 44,4 & 1,6 & 44,9 & 41,2 & 47,8 & 46,8 & 2,9 & 47,4 & 40,8 & 53,4 \\
\hline$\%$ Masa residual & 10,3 & 0,9 & 10,3 & 8,1 & 12,0 & 9,1 & 1,5 & 9,2 & 4,5 & 11,8 \\
\hline \% Masa ósea & 10,6 & 0,8 & 10,8 & 9,1 & 11,9 & 10,2 & 1,3 & 9,9 & 7,8 & 12,7 \\
\hline IMC & 22,7 & 1,0 & 22,8 & 20,4 & 24,4 & 23,4 & 2,4 & 22,8 & 20,1 & 28,4 \\
\hline ENDO & 3,9 & 1,0 & 3,7 & 2,4 & 6,1 & 3,8 & 1,4 & 3,7 & 2,0 & 6,3 \\
\hline MESO & 4,7 & 1,2 & 4,5 & 2,1 & 6,3 & 4,3 & 0,8 & 4,2 & 3,0 & 5,8 \\
\hline ECTO & 1,5 & 1,0 & 1,1 & 0,1 & 3,3 & 1,6 & 0,8 & 1,7 & 0,5 & 3,1 \\
\hline $\mathbf{X}$ & $-2,3$ & 2,0 & $-2,7$ & $-5,7$ & 0,5 & $-2,2$ & 2,0 & -2 & $-5,5$ & 0,6 \\
\hline $\mathbf{Y}$ & 4,1 & 2,4 & 3,8 & 0,1 & 7,6 & 3,2 & 1,6 & 3,7 & 0,6 & 6,8 \\
\hline
\end{tabular}

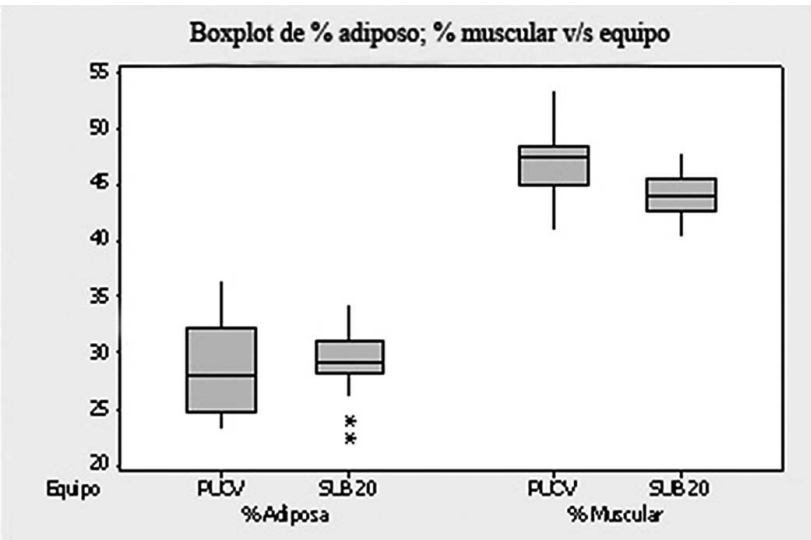

Fig. 1. Gráfico Boxplot de las medianas estadísticas y sus desvíos estándar, de masa muscular y masa adiposa, entre los equipos sub20 y equipo PUCV.

Tabla II. Tabla de las medianas estadísticas, sus desvíos estandar, y los valores de $\mathrm{p}$ del método de Krustals Wallis de las variables antropométricas entre los equipos sub 20 y equipo PUCV.

\begin{tabular}{lcrrrrrrr}
\hline \multicolumn{1}{c}{ Variable } & \multicolumn{3}{c}{ PUCV } & \multicolumn{3}{c}{ Sub 20 } & \multicolumn{3}{c}{ Valor -p } \\
& N & Mediana & $\mathbf{Z}$ & $\mathbf{N}$ & Mediana & $\mathbf{Z}$ & & \\
\hline Peso & 17 & 62,00 & 0,32 & 25 & 59,80 & $-0,32$ & 0,749 & \\
Talla & 17 & 163,00 & 1,40 & 25 & 161,00 & $-1,40$ & 0,162 & \\
IMC & 17 & 22,70 & $-0,09$ & 25 & 22,90 & 0,09 & 0,929 & \\
\% Masa adiposa & 17 & 27,94 & $-0,91$ & 25 & 29,15 & 0,91 & 0,363 & \\
\% Masa muscular & 17 & 47,43 & 3,16 & 25 & 43,92 & $-3,16$ & 0,002 & $(* *)$ \\
ENDO & 17 & 3,70 & $-0,63$ & 25 & 4,00 & 0,63 & 0,530 & \\
MESO & 17 & 4,20 & $-0,90$ & 25 & 4,50 & 0,90 & 0,369 & \\
ECTO & 17 & 1,70 & 0,95 & 25 & 1,10 & $-0,95$ & 0,342 & \\
\hline
\end{tabular}

$(* *)$ Existe diferencia significativa al $1 \%$ de significación.

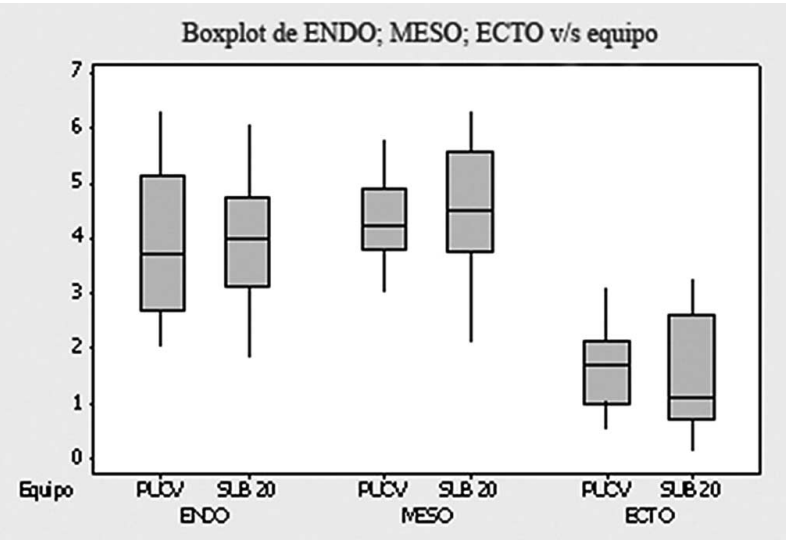

Fig. 2. Gráfico Boxplot de las medianas estadísticas y sus desvíos estándar, del somatotipo entre los equipos sub 20 y equipo PUCV. 


\section{DISCUSION}

Ambos equipos presentaron una adiposidad alta sobre otras referencias u otros deportes. Kevin Norton, en su libro “Antropométrica” (Norton \& Olds, 1996), muestra una adiposidad normal en mujeres, expresada en sumatoria de 6 pliegues entre 45 y $76 \mathrm{~mm}$. Los equipos sub 20 y PUCV presentaron una media de 82,5 y 86,5 respectivamente, considerados como condición de sobrepeso.

La clasificación del somatotipo, de acuerdo a la forma de ambos grupos al ser estadísticamente homogéneo, fue clasificado como mesoendomórfica (Kerr), con valores de 3,9-4,5-1,6 para endo, meso y ecto, respectivamente. Un estudio realizado en jugadoras de fútbol turcas (Can et al., 2004) muestra un somatotipo de 3,5-3,3-2,9, indicando una mayor robustez de las jugadoras chilenas, lo que podría desfavorecer el juego individual y favorecer el juego colectivo de ataque o defensa en contacto directo con el rival.

La alta cantidad de grasa y la robustez mayor que en otros grupos y deportes, perjudica el desempeño físico, considerando el tamaño del campo y el tiempo de juego. En un juego de 90 minutos se recorren cerca de 10 kilómetros entre el 80 y $90 \%$ de la frecuencia cardiaca máxima, lo cual resulta difícil para deportistas con exceso de peso (Polman et al., 2004). Fundamento de eso es que Mladenovic (2005) estudió los cambios ocurridos en los equipos femenino de Servia y Montenegro y demostró que al disminuir el IMC en un 3,75 y la grasa en un $1,7 \%$, se puede aumentar en un $18,4 \%$ el $\mathrm{VO}_{2}$ máx. Herrero de Lucas \& Cabañas Armesilla (
2003), en un estudio hecho en 150 futbolistas españoles de $3^{\mathrm{a}}, 2^{\mathrm{a}}$ y $1^{\mathrm{a}}$ divisiones, encontraron diferencias significativas con ANOVA en la sumatoria de 6 pliegues, los cuales disminuían a medida que aumentaba el nivel de entrenamiento.

Otro valor antropométrico importante es el de masa muscular, el cual fue significativamente mayor en el equipo PUCV que en el equipo Sub $20(\mathrm{H}=10,02 \mathrm{DF}=1 \mathrm{P}=0,002)$.

Lo anterior representa un desarrollo madurativo y deportivo aumentado, relacionado con la edad y el tiempo de actividad física, que fue mayor en el equipo PUCV debido a su condición de estudiantes de Educación Física, con una carga de entrenamiento mayor.

Las diferencias antropométricas establecidas no serían suficientes. Se puede establecer una primera referencia antropométrica comparativa para el fútbol femenino chileno juntando los dos grupos deportivos estudiados.

\section{AGRADECIMIENTOS}

Por el apoyo y financiamiento otorgados, a la Dirección de Investigación, Vice Rectoría de Investigación y Estudios Avanzados e Instituto de Biología de la Facultad de Ciencias de la Pontificia Universidad Católica de Valparaíso, Chile.

ALMAGIÀ, A. F.; RODRÍGUEZ, R. F. J.; BARRAZA, G. F. O.; LIZANA, P. J. \& JORQUERA, A. C. A. Anthropometric profile of female football - soccer chilean players. Int. J. Morphol., 26(4):817-821, 2008.

SUMMARY: A whole of 43 was evaluated of football players, 26 selected Chilean sub 20 and 17 players of the champion nursery of university football of 2007 of the Pontificia Universidad Católica de Valparaíso (PUCV), all of them under his informed assent. Was evaluated under the protocol of marking and measurement of the International Society of Avancement in Kineanthropometric (ISAK) for the in normal conditions of temperature, in the early morning and after the urinary emptying, by assessors by license level II of ISAK. The measured variables were weight, stature of foot, stature sat, muscular perimeters, bony diameters and cutaneous folds. All these measures are in use for the formulae of corporal composition of D. Kerr, (1988) and for the antropometrics method of the Somatotype of HeathCarter (2004). There exists a difference of 3, 4 years between both groups what differentiates some other components of the morphoestructure as a major quantity of oily and minor quantity of muscle of the group sub-20 in comparison with the group PUCV and a graphical representation of the somatotype that corroborates these differences. The differences in the components of the corporal composition between both groups have two fundamental reasons. The first one for the major development and physical ripeness of the group PUCV who to the major being, has averages of stature and I weigh also major. The second reason is the level of performance of the group PUCV, which on having had $\mathrm{n}$ major development allows the accomplishment of trainings them prolonged and of major intensity, demonstrating a major muscular development and minor quantity of fat in comparison with group sub-20. We conclude that according to the factors anthropometrics the group PUCV is capable to obtain a sports major performance that the group sub-20, being this a foundation to the moment to choose the national pre-selections players.

KEY WORDS: Somatotype; Body composition; Anthropometry; Female football soccer. 


\section{REFERENCIAS BIBLIOGRÁFICAS}

Can, F.; Yilmaz, I. \& Erden, Z. Morphological characteristics and performance variables of women soccer players. $J$. Strength Cond. Res.; 18(3): 480-5, 2004.

Carter, J. E. L. Factores morfológicos que limitan el rendimiento humano. Department of Kinesiology, Physical Education and Recreational Arts, San Diego State University, San Diego, California, U.S.A. publicado en español por www.sobreentrenamiento.com. Buenos Aires, 2004.

FIFA. Casi 250 millones de futbolistas en todo el mundo. Infoplus.http//images.fifa.com/images/pdf/IP499_01E_BigCount.http.

Herrero de Lucas, A. \& Cabañas Armesilla. Evaluación comparativa de la distribución corporal de tejido adiposo entre jugadores de fútbol profesionales, semiprofesionales y amateurs. Biomecánica, 11:23-9, 2003.

Kerr, D. A. An anthropometric method for the fracitionation of skin, adipose, muscle, bone and residual tissue masses in males and females age 6 to 77 years. M. Sc. Thesis. Simon Fraser University, 1988.

Mladenovic, I. Developing Characteristics and functional habilitéis of top female football platers. J. Medicine and Biology, 12(2):97-9, 2005.

Norton, K. \& Olds, T. Antropometrica. Southwood Press, Marrickville, Sidney, 1996.

Polman, R.; Walsh D.; Blonmfield, J. \& Mesti, M., Effective conditioning of female soccer player. J. Sports Sci.; 22:191-203, 2004.
Dirección para correspondencia:

Prof. Atilio Aldo Almagià Flores

Avda. Brasil 2950

Casilla 4059

Pontificia Universidad Católica de Valparaíso

Valparaíso

CHILE

Fax: 56-32-212746

Email: aalmagia@ucv.cl

Recibido : 16-09-2008

Aceptado: 22-10-2008 